\title{
Relativistic models for Superconducting-Superfluid Mixtures
}

\section{Brandon Carter and David Langlois}

\author{
Département d'Astrophysique Relativiste et de Cosmologie, \\ Centre National de la Recherche Scientifique, \\ Observatoire de Paris, 92195 Meudon, France.
}

\section{May 1998}

\begin{abstract}
The material below the crust of a neutron star is understood to be describable in terms of three principal independently moving constituents, identifiable as neutrons, protons, and electrons, of which the first two are believed to form mutually coupled bosonic condensates. The large scale comportment of such a system will be that of a positively charged superconducting superfluid in a negatively charged "normal" fluid background. As a contribution to the development of the theory of such a system, the present work shows how, subject to neglect of dissipative effects, it is possible to set up an elegant category of simplified but fully relativistic three-constituent superconducting superfluid models whose purpose is to provide realistic approximations for cases in which a strictly conservative treatment is sufficient. A "mesoscopic" model, describing the fluid between the vortices, is constructed, as well as a "macroscopic" model taking into account the average effect of quantised vortices.
\end{abstract}




\section{Introduction}

According to the generally accepted understanding, apart from an outer magnetosphere of relatively negligible mass and perhaps also, in the most massive examples, of an inner core consisting of matter in some more or less exotic state at more than nuclear density, a neutron star can be described in terms of three principle layers: as well as the degenerate gas of electrons (and muons) that is present throughout, the outside layer consists of a "dry" solid upper crust formed just by a lattice of positively charged ions in a degenerate electron gas; the middle layer consists of a "wet" lower crust, whose solid ionic lattice is interpenetrated by a neutron superfluid; finally the interior region consists of a neutron superfluid in which the ionic lattice has dissolved to form a superconducting proton fluid. The purpose of the present article is to develop a simplified model that can be used to represent the dynamics of this superconducting superfluid interior, which constitutes the greater part of the mass of a typical middlesized neutron star.

For purposes for which only a very crude description of the bulk motion of the interior superconducting superfluid region is needed, it may be sufficient to use a model involving only two independently moving constituents, of which one represents the neutron superfluid while the other represents everything else, since short range electromagnetic interactions will tend to ensure that the positively charged protons and the negatively charged electrons will tend to move together as an approximately rigid background. One of the advantages of such a two constituent description is that it is also applicable to the crust layers, so that - in an approximation in which details such as the small anisotropies due to the solidity of the crust are neglected - it can be used globally to provide a continuously unified description of the star as a whole. A suitable two-constituent fluid model for this purpose has recently been constructed[1] in a relativistic form that includes allowance for "transfusion", meaning the slow transfer of baryonic matter (microscopically attributable to processes such as beta-decay of neutrons to protons) between the neutron superfluid and the other "normal" constituent.

Although adequate for many purposes, the two-constituent fluid treatment [1] that has just been alluded to is insufficient for the detailed description of phenomena such as rotation frequency "glitches" - which are observable via the pulsar phenomena - that depend on the small deviations from isotropy that occur not only in the crust (as a consequence of its solidity) but also in the interior, due to the effect of vorticity quantisation and also (at least in the cases that are observable via the pulsar phenomena) to the presence of large scale magnetic fields. Using an variational approach based on the use of a Kalb-Ramond gauge potential in the manner originally introduced for the treatment of ordinary barotropic perfect fluids [2], the present authors have previously shown[3] how to construct a kind of model that - at least over short and moderate timescales for which "transfusion" processes can be neglected - can provide a satisfactorily relativistic macroscopic description of the effect of the anisotropy due to fibration by quantised vortices in what is describable, at a "mesoscopic" (submacroscopic but more than microscopic) scale outside the vortices, by a simple superfluid model. However although this kind of anisotropic superfluidity model is directly applicable to the 
terrestrially familiar example of liquid helium, the utility for neutron stars of such a single-constituent representation is limited by its neglect of the electromagnetically interacting constituents, namely the electrons and the protons, which together represent only a small fraction of the mass density, but which nevertheless play an essential role in phenomena involving magnetic effects.

It has already been shown that the variational treatment in terms of Kalb-Ramond potentials can be straightforwardly generalised from the original single-constituent perfect fluid case 2] to the case of perfect two-constituent electrically conducting fluid models [4] (including the special kind - with the negatively charged constituent restricted to be irrotational - that can be used for a "mesoscopic" description of the experimentally familiar kind of type II electronic superconductor using a crude but fully relativistic representation that neglects the anisotropy due to the solid, not fluid, structure of the positively charged ionic background constituent). Following this example, the purpose of the present work is to show how - still neglecting the "transfusion" processes that are likely to be important only in the very long run - our previous macroscopic model[3], representing the effect of vortex fibration in a simple superfluid, can be extended to a three-constituent model providing a description that is applicable to a superconducting superfluid in which the other two independently moving constituents are the superfluid protons and the degenerate electron gas.

A considerable amount of work on three-constituent models for this purpose has already been carried out by several authors [5, 6, 7, 8, but until now only within the framework of a "non-relativistic" Newtonian description (as a generalisation of the kind of two-constituent model used for treatment of an ordinary "type II" metallic superconductors of the sort familiar in a laboratory context, for which the two constituents are superconducting electrons and a solid ionic background lattice). Some of this previous work includes allowance for details such as dissipative effects e.g. to various kinds of resistivity, that in the present description will for the sake of clarity will be provisionally left aside for treatment in a subsequent treatment. The omission of such dissipative effects is however by no means the only reason why the conservative treatment given here turns out to be technically simpler and more elegant than its "non-relativistic" predecessors: the other reason is that the Galilei invariance group applicable to the Newtonian description is more complicated than (since it is a degenerate limit of) the (in the technical sense "semi-simple") Lorentz invariance group that is locally applicable to the fully (general as well as special) relativistic treatment provided here. When the electromagnetic coupling is taken into account, the relativistic description is even more satisfying in that the material part and the electromagnetic part are treated on the same footing instead of the awkward mixture of Galilean and Lorentzian invariances in the Newtonian context.

It is to be remarked that the improvement not just in physical accuracy but also in mathematical elegance that is obtainable by going from a Newtonian to a relativistic treatment has already been demonstrated in the case of Landau's original twoconstituent superfluid model (in which the second constituent just represents the entropy current), whose relativistic generalisation 9.9 has provided insights that have lead 
to a more efficient "canonical" description [10] of its original Newtonian version. This suggests, as a challenge for future work, that the traditional presentation of the more elaborate three-constituent fluid models developed in previous work [6, 7, 8], should also be susceptible to similar improvements in elegance by an analogous "canonical" reformulation along lines that would presumably be obtainable by taking an appropriate Newtonian limit of the even more elegant relativistic kind of model developed here.

Of greater importance than the analysis of the Newtonian limit, as a challenge for future work, the kind of model developed here will need to be completed by the provision (on the basis of appropriate microphysical analysis) of explicit forms and values for the functions and parameters involved, while it will also be necessary to develop the more extended treatment needed to include suitable allowance for dissipative effects (not to mention refinements such as allowance for the spinor as opposed to scalar nature of the Cooper pairs involved in the microscopic description 11] of both the neutron superfluid and the superconducting proton current).

Another challenge is the development of a corresponding relativistic description for the solid crust layers. An appropriate relativistic formalism for describing the purely solid "dry" upper crust layer of a Newton star was developed quite a long time ago 12, 13, 14, though its technical complexity has delayed its effective application to specific astrophysical problems[15]. What has yet to be constructed is the even more elaborate generalisation required for treating the "wet" lower layer of the solid crust: machinery capable of providing a treatment on a "mesoscopic" scale of the interaction between the permeating neutron superfluid and the solid background lattice has already been made available[16], but it still remains to develop the corresponding macroscopic analysis required for the relativistic description of the large scale effects of phenomena such as vortex pinning[17] in such a "wet" solid.

As declared above, the ultimate task of the present work is to provide a kind of model that can account for the macroscopic anisotropies attributable to quantised vortex lines associated with both rotation and magnetic fields, in the deep superconducting superfluid interior of a neutron star. However, as a preliminary step, we must first develop a suitably relativistic "mesoscopic" (submacroscopic but not microscopic) scale treatment applicable to the superconducting superfluid in between the relevant stringlike vortex defects. The most elegant versions of the models presented below are those that apply to the zero temperature limit, which should be a good enough approximation for most relevant astrophysical applications in neutron stars. We shall however include a rudimentary allowance for the effect of a finite temperature associated with a non-vanishing conserved entropy distribution comoving with the degenerate electrons in the negatively charged "normal" constituent.

\section{Three constituent perfectly conducting fluids.}

Following what has long been established as a routine procedure 18 - (the natural relativistic analogue of the phenomenological approach developed in a Newtonian context 
[19] as a generalisation of Landau's original two-constituent model) - the task of the present section is to set up the category of simple three-constituent perfectly conducting fluid models that is needed as a preliminary basis for the more specific developments that follow. As the specialisation to be treated in the next section, this category includes the particular restricted case that is appropriate for the treatment of the kind of superconducting superfluid that is relevant in neutron stars on a "mesoscopic" scale, meaning a scale large compared with that of the underlying microscopic particle description, but small compared with the macroscopic scale of separation between the vortex defects on which the superfluid comportment is locally violated. On the other hand the category set up in the present section is itself a just specially simple limit within the more general category to be developed in the later sections for the purpose of treating the superconducting superfluid on a "macroscopic" scale, meaning a scale that is large compared with the separation between vortices.

The three independent constituents under consideration are identifiable as the superfluid neutrons - which make up most of the mass density in the relevant neutron star layers - with number current four-vector $n_{\mathrm{n}}^{\rho}$, the superconducting protons, - which make up a small but significant part of the mass density - with number current fourvector $n_{\mathrm{p}}^{\rho}$, and finally the degenerate non-superconducting background of electrons with number current vector $n_{\mathrm{e}}^{\rho}$. The latter make up a negligibly small fraction of the mass density, but they nevertheless have a crucially important role in so far as electomagnetic effects are concerned, since in terms of the electron charge coupling constant $e$ the corresponding total electric current vector four vector will be given by

$$
j^{\mu}=e\left(n_{\mathrm{p}}^{\rho}-n_{\mathrm{e}}^{\rho}\right) .
$$

As well as the three principal constituents that have just been listed, our discussion in this section will also include allowance for a fourth constituent, namely the entropy. However this will not be treated as fully independent, since (as will be a realistic approximation for most of the relevant astrophysical applications) its current fourvector $s^{\mu}$ will be postulated to be constrained so as to be aligned with that of the electrons. This means that, apart from the "super" constituents with currents $n_{\mathrm{n}}^{\rho}$ and $n_{\mathrm{p}}^{\rho}$, there will be just a single independently moving "normal" constituent with current in the direction specified by a unit vector $u^{\rho}$, as characterised by the normalisation condition

$$
u^{\rho} u_{\rho}=-1,
$$

(on the usual understanding that the units are such that the speed of light is unity) with

$$
\begin{gathered}
n_{\mathrm{e}}^{\rho}=n_{\mathrm{e}} u^{\rho}, \\
s^{\rho}=s u^{\rho},
\end{gathered}
$$

where the scalars $s$ and $n_{\mathrm{e}}$ are respectively the entropy density and the electron number density in the "normal" rest frame specified by $u^{\rho}$.

Since our present treatment will be restricted to the conservative limit in which dissipative effects are neglected, the analysis will be assumed to be expressible in terms 
of a variational principle based on a Lagrangian density that, in the absence of electromagnetic effects, can be presumed to be given by a function $\Lambda_{\mathrm{M}}$ say depending just on the six independent scalars obtainable by mutual contractions of the three independent current vectors $n_{\mathrm{n}}^{\rho}, n_{\mathrm{p}}^{\rho}, n_{\mathrm{e}}^{\rho}$ and on the entropy density $s$. The independent variations of these quantities determine the effective momentum covectors $\mu_{\mu}^{\mathrm{n}}$ and $\mu_{\mu}^{\mathrm{p}}$ respectively associated with the superfluid neutrons and the superconducting protons, together with the "normal" momentum covector $\mu_{\mu}^{\mathrm{e}}$ associated with the electrons, and the corresponding temperature $\Theta$, via a prescription of the form

$$
\delta \Lambda_{\mathrm{M}}=\mu_{\rho}^{\mathrm{n}} \delta n_{\mathrm{n}}^{\rho}+\mu_{\rho}^{\mathrm{p}} \delta n_{\mathrm{p}}^{\rho}+\mu_{\rho}^{\mathrm{e}} \delta n_{\mathrm{e}}^{\rho}-\Theta \delta s+\frac{\partial \Lambda_{\mathrm{M}}}{\partial g_{\rho \sigma}} \delta g_{\rho \sigma},
$$

in which, as an elementary Noether type identity, we shall automatically have

$$
\frac{\partial \Lambda_{\mathrm{M}}}{\partial g_{\rho \sigma}}=\frac{\partial \Lambda_{\mathrm{M}}}{\partial g_{\sigma \rho}}=\frac{1}{2}\left(\mu_{\nu}^{\mathrm{n}} n_{\mathrm{n}}^{\rho}+\mu^{\mathrm{p}}{ }_{\nu} n_{\mathrm{p}}^{\rho}+\mu^{\mathrm{e}}{ }_{\nu} n_{\mathrm{e}}^{\rho}\right) g^{\nu \sigma} .
$$

It is useful to allow here for the possibility of varying the background spacetime metric $g_{\rho \sigma}$, not only for the purpose of dealing with cases in which one may be concerned with General Relativistic gravitational coupling, but even for dealing with cases in which one is concerned only with a flat Minkowski background, since, as will be made explicit below, the effect of virtual variations with respect to the relevant curved or flat background metric can be used for evaluating the relevant "geometric" stress energy momentum density tensor $T^{\rho \sigma}$.

The preceding formulae (5) and (6) can conveniently be combined in a single expression of the more concise form

$$
\delta \Lambda_{\mathrm{M}}=\mu_{\rho}^{\mathrm{x}} \delta n_{\mathrm{x}}^{\rho}-\Theta \delta s+\frac{1}{2} \mu_{\nu}^{\mathrm{x}} n_{\mathrm{x}}^{\rho} g^{\nu \sigma} \delta g_{\rho \sigma},
$$

using the summation convention for "chemical" indices represented by capital Latin letters running over the three relevant values, namely $\mathrm{x}=\mathrm{n}, \mathrm{p}, \mathrm{e}$. Using this convention, the equation (11) for the electric current density can be rewritten in the concise form

$$
j^{\rho}=e^{\mathrm{x}} n_{\mathrm{x}}^{\rho}
$$

where the charges per neutron, proton, and electron are given respectively by $e^{\mathrm{n}}=0$, $e^{\mathrm{p}}=e$, and $e^{\mathrm{e}}=-e$.

The standard minimal prescription for inclusion of electromagnetic interactions is to use a combined Lagrangian scalar density in which the "matter" contribution $\Lambda_{\mathrm{M}}$ is augmented by an electromagnetic field contribution $\Lambda_{\mathrm{F}}$ and a gauge dependent coupling term of the usual form to give a total Lagrangian scalar $\mathcal{L}$ expressible as

$$
\mathcal{L}=\Lambda+j^{\rho} A_{\rho}
$$

where the gauge independent part has the form

$$
\Lambda=\Lambda_{\mathrm{M}}+\Lambda_{\mathrm{F}},
$$


and where $A_{\rho}$ is the electromagnetic gauge form, while the gauge independent contribution $\Lambda_{\mathrm{F}}$ is given in terms of the corresponding electromagnetic field tensor,

$$
F_{\rho \sigma}=2 \nabla_{[\rho} A_{\sigma]}
$$

(using square brackets to indicate index antisymmetrisation) by the standard Maxwellian formula

$$
\Lambda_{\mathrm{F}}=\frac{1}{16 \pi} F_{\rho \sigma} F^{\sigma \rho} .
$$

The variation of the total will then be given by

$$
\delta \mathcal{L}=\pi_{\rho}^{\mathrm{x}} \delta n_{\mathrm{x}}^{\rho}-\Theta \delta s+j^{\rho} \delta A_{\rho}+\frac{1}{8 \pi} F^{\sigma \rho} \delta F_{\rho \sigma}+\frac{1}{2}\left(\mu^{\mathrm{x}}{ }_{\nu} n_{\mathrm{x}}^{\rho} g^{\nu \sigma}+\frac{1}{4 \pi} F^{\nu \rho} F_{\nu}{ }^{\sigma}\right) \delta g_{\rho \sigma},
$$

where the gauge dependent total momentum covectors are given by

$$
\pi_{\rho}^{\mathrm{x}}=\mu_{\rho}^{\mathrm{x}}+e^{\mathrm{x}} A_{\rho} .
$$

In order to characterise a variation principle that can be used to specify the equations of motion of a multiconstituent medium, the electromagnetic potential covector $A_{\rho}$ is of course allowed to vary freely, but the variations of the independent currents $n_{\mathrm{x}}^{\rho}$ cannot be allowed to be arbitrary, since this would evidently lead to an overdetermined system in which the momenta would simply have to vanish. The standard procedure for restricting the allowed current variations so as to obtain a system with the appropriate number of degrees of freedom for an ordinary fluid mixture is to require that the the current variations be determined by free displacements of the corresponding worldlines - in the manner originally introduced for the particular case of a relativistic perfect fluid by Taub 20]. The ensuing variational equations then automatically ensure the conservation under transport by each current flow $n_{\mathrm{x}}^{\rho}$ of the corresponding generalised vorticity tensor as defined by

$$
w_{\rho \sigma}^{\mathrm{x}}=2 \nabla_{[\rho} \pi_{\sigma]}^{\mathrm{x}}=2 \nabla_{[\rho} \mu_{\sigma]}^{\mathrm{x}}+e^{\mathrm{x}} F_{\rho \sigma} .
$$

Such an exterior derivative will of course automatically be "closed", i.e. its own exterior derivative will vanish:

$$
\nabla_{[\nu} w_{\rho \sigma]}^{\mathrm{x}}=0 .
$$

Within such a perfect multiconstituent fluid category, the special properties of superfluidity and superconductivity are characterised by the constraint that the corresponding conserved vorticity should vanish initially, and hence, since it is conserved, throughout the ensuing evolution of the system. Such constraints - which in our present application concern the neutrons and the protons but not the electrons - are interpretable as integrability conditions for corresponding potential scalars. For the neutrons we shall have

$$
w_{\rho \sigma}^{\mathrm{n}}=0 \Rightarrow \pi_{\rho}^{\mathrm{n}} \equiv \mu_{\rho}^{\mathrm{n}}=\frac{\hbar}{2} \nabla_{\rho} \varphi^{\mathrm{n}}
$$


while for the protons we shall have

$$
w_{\rho \sigma}^{\mathrm{p}}=0 \Rightarrow \pi_{\rho}^{\mathrm{p}} \equiv \mu_{\rho}^{\mathrm{p}}+e A_{\rho}=\frac{\hbar}{2} \nabla_{\rho} \varphi^{\mathrm{p}}
$$

where the locally defined potentials $\varphi^{\mathrm{n}}$ and $\varphi^{\mathrm{p}}$ are interpretable as phase angles (with period $2 \pi$ ) associated with underlying boson condensates, and the factors 2 in the denominators are included to allow for the fact that the relevant bosons are presumed to consist not of single protons or neutrons but of Cooper type pairs.

The way the Taub type "convective" variational procedure works out is as follows. For any current vector $n^{\rho}$ the corresponding variation rule is interpretable as meaning that the ensuing variation of its dual three-form, as given - in terms of the antisymmetric measure tensor $\varepsilon_{\mu \nu \rho \sigma}$ associated with the 4-dimensional spacetime metric $g_{\mu \nu}$ - by

$$
{ }^{*} n_{\mu \nu \rho}=\varepsilon_{\mu \nu \rho \sigma} n^{\sigma}
$$

should be specified by its Lie derivative with respect to the relevant worldline displacement vector field, $\xi^{\rho}$ say, i.e.

$$
\delta^{*} n_{\mu \nu \rho}=\xi^{\sigma} \nabla_{\sigma}^{*} n_{\mu \nu \rho}+3^{*} n_{\sigma[\mu \nu} \nabla_{\rho]} \xi^{\sigma}
$$

where $\nabla_{\mu}$ denotes the covariant differentiation operator specified by the relevant (flat or curved) spacetime background metric $g_{\mu \nu}$.

When applied to the particular case of the "normal" (i.e. non-superfluid) electron current $n_{\mathrm{e}}^{\rho}$, for a corresponding displacement vector field $\xi_{\mathrm{e}}^{\rho}$, the foregoing prescription can be seen to give a variation of the form

$$
\delta n_{\mathrm{e}}^{\rho}=\xi_{\mathrm{e}}^{\sigma} \nabla_{\sigma} n_{\mathrm{e}}^{\rho}-n_{\mathrm{e}}^{\sigma} \nabla_{\sigma} \xi_{\mathrm{e}}^{\rho}+n_{\mathrm{e}}^{\rho} \nabla_{\sigma} \xi_{\mathrm{e}}^{\sigma}-\frac{1}{2} n_{\mathrm{e}}^{\rho} g^{\mu \nu} \delta g_{\mu \nu},
$$

from which it follows that the variation of the scalar electron number density introduced in (3) will be given by

$$
\delta n_{\mathrm{e}}=\xi_{\mathrm{e}}^{\sigma} \nabla_{\sigma} n_{\mathrm{e}}+n_{\mathrm{e}} u_{\rho} u^{\sigma} \nabla_{\sigma} \xi_{\mathrm{e}}^{\rho}+n_{\mathrm{e}} \nabla_{\sigma} \xi_{\mathrm{e}}^{\sigma}-\frac{1}{2} n_{\mathrm{e}}\left(g^{\mu \nu}+u^{\mu} u^{\nu}\right) \delta g_{\mu \nu}
$$

Since we are postulating that the electron current is comoving with the entropy current as part of a combined "normal" constituent, for which the shared unit 4-vector appearing in (3) and (4) will thus be subject to the variation law

$$
\delta u^{\rho}=\xi_{\mathrm{e}}^{\sigma} \nabla_{\sigma} u^{\rho}-u^{\sigma} \nabla_{\sigma} \xi_{\mathrm{e}}^{\rho}-u^{\rho} u_{\nu} u^{\sigma} \nabla_{\sigma} \xi_{\mathrm{e}}^{\nu}+\frac{1}{2} u^{\rho} u^{\mu} u^{\nu} \delta g_{\mu \nu}
$$

it follows that the variation of the entropy current will be governed by the same displacement vector field $\xi_{\mathrm{e}}^{\rho}$ as that of the electron current, and hence the variation of the entropy density introduced in (四) will be governed by a rule of the same form as (22), namely

$$
\delta s=\xi_{\mathrm{e}}^{\sigma} \nabla_{\sigma} s+s u_{\rho} u^{\sigma} \nabla_{\sigma} \xi_{\mathrm{e}}^{\rho}+s \nabla_{\sigma} \xi_{\mathrm{e}}^{\sigma}-\frac{1}{2} s\left(g^{\mu \nu}+u^{\mu} u^{\nu}\right) \delta g_{\mu \nu}
$$


When metric variations are involved, the application of the variation principle requires allowance not just for the variation of the Lagrangian scalar $\mathcal{L}$, but also for the variation of the relevant spacetime measure, which is proportional to the square root of the modulus of the metric determinant $|g|$. This means that the complete variational integrand that one needs to evaluate is the "diamond" variation as defined by

$$
\diamond \mathcal{L}=\|g\|^{-1 / 2} \delta\left(\|g\|^{1 / 2} \mathcal{L}\right)=\delta \mathcal{L}+\frac{1}{2} \mathcal{L} g^{\mu \nu} \delta g_{\mu \nu}
$$

By taking the variations of the neutron and proton currents to be given in the same way as that of the electron current by corresponding displacement vector fields $\xi_{\mathrm{n}}^{\rho}$ and $\xi_{\mathrm{p}}^{\rho}$, the required "diamond" variation of the Lagrangian scalar density given by (9) can be obtained in the standard form

$$
\diamond \mathcal{L}=f_{\rho}^{\mathrm{x}} \xi_{\mathrm{X}}^{\rho}+\left(j^{\rho}-\frac{1}{4 \pi} \nabla_{\sigma} F^{\rho \sigma}\right) \delta A_{\rho}+\frac{1}{2} T^{\mu \nu} \delta g_{\mu \nu}+\nabla_{\sigma} \mathcal{R}^{\sigma}
$$

in which the coefficients $f_{\rho}^{\mathrm{x}}$ of the independent displacement displacement vector fields $\xi_{\mathrm{x}}^{\rho}$ are respectively interpretable as the force densities acting on the corresponding constituents, while the (symmetric) tensor $T^{\mu \nu}$ evidently represents the ordinary ("geometric") stress momentum energy density. It can be seen that the force densities acting on the superfluid neutrons and the superconducting protons will be given respectively by

$$
f_{\rho}^{\mathrm{n}}=n_{\mathrm{n}}^{\sigma} w_{\sigma \rho}^{\mathrm{n}}+\mu_{\rho}^{\mathrm{n}} \nabla_{\sigma} n_{\mathrm{n}}^{\sigma}
$$

and

$$
f_{\rho}^{\mathrm{p}}=n_{\mathrm{p}}^{\sigma} w_{\sigma \rho}^{\mathrm{p}}+\pi_{\rho}^{\mathrm{p}} \nabla_{\sigma} n_{\mathrm{p}}^{\sigma} .
$$

where $w_{\sigma \rho}^{\mathrm{n}}$ and $w_{\sigma \rho}^{\mathrm{p}}$ are the corresponding vorticity two-forms as defined by (15). The force density acting on the "normal" constituent - consisting of the electrons and the comoving entropy current - can be seen to be given by an expression of the not quite so simple form

$$
f_{\rho}^{\mathrm{e}}=2 u^{\sigma} \nabla_{[\sigma} \Pi_{\rho]}+\Pi_{\rho} \nabla_{\sigma} u^{\sigma}+u^{\sigma} \pi_{\sigma}^{\mathrm{e}} \nabla_{\rho} n_{\mathrm{e}}-\Theta \nabla_{\rho} s
$$

in which the one-form $\Pi_{\rho}$ is the effective momentum density of the normal constituent (electrons and entropy) treated as a whole, as given by

$$
\Pi_{\rho}=n_{\mathrm{e}} \pi_{\rho}^{\mathrm{e}}+s \Theta u_{\rho}
$$

The stress momentum energy density tensor can be seen to have the form

$$
T^{\rho \sigma}=T_{\mathrm{M}}^{\rho \sigma}+T_{\mathrm{F}}{ }^{\rho \sigma},
$$

where the part derived just from the material Lagrangian density contribution $\Lambda_{\mathrm{M}}$ is given by

$$
T_{\mathrm{M} \sigma}^{\rho}=n_{\mathrm{x}}^{\rho} \mu_{\sigma}^{\mathrm{x}}+s \Theta u^{\rho} u_{\sigma}+\Psi_{\mathrm{M}} g_{\sigma}^{\rho}, \quad \Psi_{\mathrm{M}}=\Lambda_{\mathrm{M}}-n_{\mathrm{x}}^{\sigma} \mu_{\sigma}^{\mathrm{x}}+s \Theta
$$


while the electromagnetic contribution has the usual Maxwellian form

$$
T_{\mathrm{F}}^{\rho}{ }_{\sigma}=\frac{1}{4 \pi}\left(F^{\nu \rho} F_{\nu \sigma}-\frac{1}{4} F^{\mu \nu} F_{\mu \nu} g_{\sigma}^{\rho}\right) .
$$

Although it is irrelevant as far as the variation principle is concerned, it is to be noted for the sake of completeness that the remainder current in the final divergence term of (26) will be given by

$$
\mathcal{R}^{\sigma}=2 \pi_{\nu}^{\mathrm{x}} \xi_{\mathrm{x}}^{[\sigma} n_{\mathrm{x}}^{\nu]}+2 \Theta u_{\nu} \xi_{\mathrm{e}}^{[\sigma} s^{\nu]}+\frac{1}{4 \pi} F^{\nu \sigma} \delta A_{\nu}
$$

It is evident from (26) that the requirement of invariance of the action with respect to free variations of the gauge form $A_{\rho}$ leads to electromagnetic source equations of the usual Maxwellian form

$$
\nabla_{\sigma} F^{\rho \sigma}=4 \pi j^{\rho} .
$$

Subject to this condition, it follows as a Noether type identity (obtainable by identifying all the displacement vectors with a single vector field with respect to which the system is displaced as a whole 16, 21]) that, independently of the other field equations one must have a force balance relation of the form

$$
f_{\rho}^{\mathrm{n}}+f_{\rho}^{\mathrm{p}}+f_{\rho}^{\mathrm{e}}=\nabla_{\sigma} T_{\rho}^{\sigma},
$$

in which the right hand side represents the total force density acting on the medium, which will of course vanish for the kind of isolated strictly conservative model with which we are concerned here. More particularly, it is evident from (26) that the content of the Taub type "convective" variation principle expressed by the condition that independent adjustments of the displacement fields should have no effect is equivalent to the dynamical requirement that each of the separate force density contributions on the left of (36) should vanish separately: in short the equations of motion of the system will be expressible simply by the condition

$$
f_{\rho}^{\mathrm{x}}=0
$$

for each of the three relevant chemical index values $\mathrm{X}=\mathrm{n}, \mathrm{p}$,e.

Assuming that (unlike what would be required for a model of "transfusive" type[1) there is no algebraical constraint on the momenta, it can be seen (by contracting the relevant force formulae (27) and (28) with the corresponding current vectors) that for the cases of the neutrons and the protons the conditions (37) include the implication that their currents are separately conserved,

$$
\nabla_{\rho} n_{\mathrm{n}}^{\rho}=0, \quad \nabla_{\rho} n_{\mathrm{p}}^{\rho}=0 .
$$

The remaining contents of the neutron and proton equations of motion will simply reduce to the respective forms

$$
n_{\mathrm{n}}^{\sigma} w_{\sigma \rho}^{\mathrm{n}}=0, \quad n_{\mathrm{p}}^{\sigma} w_{\sigma \rho}^{\mathrm{p}}=0
$$


where the vorticity two forms are as defined by (15). It is well known that the form of such equations ensures that, in view of the closure property (16), the neutron and proton vorticity forms will be conserved in the sense of being dragged along by the corresponding flows [16], which means that they are consistent with the special requirements for superfluidity and superconductivity respectively, which are that they should simply vanish, in accordance with (17) and (18).

For the non-superfluid "normal" constituent, the implications of the zero force condition (37) are not so simple. In this case, it can be seen from (29) that contraction with the relevant unit flow tangent vector will just give

$$
u^{\rho} \pi_{\rho}^{\mathrm{e}} \nabla_{\sigma} n_{\mathrm{e}}^{\sigma}=\Theta \nabla_{\sigma} s^{\sigma}
$$

To obtain separate current conservation laws that are required, it should be understood that the electron current is not specified freely but is given by a procedure of the kind appropriate for non-barotropic perfect fluid theory 22 via a projection (of the kind that is standard as the basis of relativistic elasticity theory [12, [13]) onto a three dimensional base manifold endowed with its own measure. This means that in terms of the spacetime pull-backs of suitably chosen base coordinates $X^{A}, A=1,2,3$, it will be given, using the abbreviation $X_{\mu}^{A}=\nabla_{\mu} X^{A}$, by an expression of the form $n_{\mathrm{e}}^{\mu}=\varepsilon^{\mu \nu \rho \sigma} X_{{ }_{\nu}}{ } X^{2}{ }_{, \rho} X^{3}{ }_{, \sigma}$. The variation law (21) is equivalent to what is obtained by allowing free variations of the $X^{A}$ considered as a set of three independent scalar fields on spacetime: such variations are equivalent to the specification of a corresponding displacement vector field $\xi_{\mathrm{e}}^{\rho}$ for which one will have $\delta X^{A}=\xi_{\mathrm{e}}^{\rho} \nabla_{\rho} X^{\rho}$. Such a construction automatically ensures that electron current will be identically conserved, and the dynamical equation (40) then entails a similar condition for the entropy, i.e. one ends up with the separate conservation laws

$$
\nabla_{\sigma} n_{\mathrm{e}}^{\sigma}=0, \quad \nabla_{\sigma} s^{\sigma}=0 .
$$

Under these conditions, the remaining contents of the equations of motion of the "normal" constituent, as expressed by the vanishing according to (37) of the force density $f_{\rho}^{\mathrm{e}}$ given by (29), will take the form

$$
n_{\mathrm{e}}^{\sigma} w_{\sigma \rho}^{\mathrm{e}}+s^{\sigma} \nabla_{\sigma}\left(\Theta u_{\rho}\right)+s \nabla_{\rho} \Theta=0
$$

which - unlike the corresponding dynamical equations (39) for the neutrons and the protons - does not have the form of a generalised vorticity conservation law, except in the zero temperature limit, $\Theta=0$, for which only the first term remains.

\section{Mesoscopic superconducting superfluid model.}

Within the category of perfectly conducting fluid motions, as characterised by the equations of motion (38), (39), (41), (42), the "mesoscopic" superconducting superfluid case relevant on an intermediate scale (in between the vortices) will be characterised by the constraints (17) and (18), whose compatibility with the dynamical equations is 
ensured by the particular form of (38). Instead of obtaining them as a particular case within the general perfectly conducting fluid category, we can proceed directly to the specialised superconducting superfluid equations of motion, as given just by (38), (41), (42) together - in accordance with (17) and (18) - with

$$
2 \pi_{\rho}^{\mathrm{n}}=\hbar \nabla_{\rho} \varphi^{\mathrm{n}}, \quad 2 \pi_{\rho}^{\mathrm{p}}=\hbar \nabla_{\rho} \varphi^{\mathrm{p}}
$$

by using a variational procedure of a modified kind. Since this new kind of procedure will treat the "super" currents on a rather different footing from the others, its presentation will be facilitated by the introduction of a correspondingly restricted chemical index, for which we shall use the capital Greek letters $\Upsilon, \Phi$ which differ from the unrestricted chemical indices $X, Y$ in having a range that is restricted to exclude the "normal" current values. For the three-constituent category under consideration here, the only "normal" value to be excluded is $X=\mathrm{e}$. It follows that, for this category, the Greek letters will be interpretable as isotopic indices, taking the pair of values $\Upsilon=\mathrm{n}$ for the neutron case and $\Upsilon=\mathrm{p}$ for the proton case. With this convention, the two separate equations (43) can be combined into the more concise form

$$
2 \pi_{\rho}^{r}=\hbar \nabla_{\rho} \varphi^{r}
$$

in which it is to be recalled that the factor 2 is present in order that the left hand side should represent the effective momentum covector of the relevant bosonic unit, which will consist not of a single baryon but of a Cooper type pair.

According to the rules of the modified procedure, instead of being restricted to satisfy displacement variation rules of the kind (21) satisfied by the "normal" constituent, it is permissible for the isotopic doublet formed by the "super" currents $n_{\mathrm{n}}^{\rho}$ and $n_{\mathrm{p}}^{\rho}$ to vary freely apart from the restriction that the corresponding conservation laws (38) should be satisfied. The enforcement of these conservation laws, which can be combined in the single expression

$$
\nabla_{\sigma} n_{\Upsilon}^{\sigma}=0
$$

is to be obtained in the usual way by the introduction of corresponding Lagrange multipliers. Provided they are introduced with the appropriate normalisation, these multipliers turn out to be identifiable with the potentials $\varphi^{\mathrm{n}}$ and $\varphi^{\mathrm{p}}$ introduced in (17) and (18). This is done my making the replacement

$$
\mathcal{L} \mapsto \mathcal{L}_{\mathrm{I}}
$$

where the modified Lagrangian scalar is given by the expression

$$
\mathcal{L}_{\mathrm{I}}=\mathcal{L}+\frac{\hbar}{2} \varphi^{\Upsilon} \nabla_{\sigma} n_{\Upsilon}^{\sigma}
$$

in which summation over the isotopic index $\Upsilon$ is of course to be understood. The correspondingly modified replacement of the "diamond" variation formula (26) is 


$$
\begin{aligned}
& \diamond \mathcal{L}_{\mathrm{I}}=(\left.\pi_{\rho}^{\Upsilon}-\frac{\hbar}{2} \nabla_{\rho} \varphi^{\Upsilon}\right) \delta n_{\Upsilon}^{\rho}+f_{\rho}^{\mathrm{e}} \xi_{\mathrm{e}}^{\rho}+\left(j^{\rho}-\frac{1}{4 \pi} \nabla_{\sigma} F^{\rho \sigma}\right) \delta A_{\rho} \\
&+\frac{\hbar}{2}\left(\nabla_{\sigma} n_{\Upsilon}^{\sigma}\right) \delta \varphi^{\Upsilon}+\frac{1}{2} T^{\mu \nu} \delta g_{\mu \nu}+\nabla_{\sigma} \mathcal{R}_{\mathrm{I}}^{\sigma},
\end{aligned}
$$

in which the force density $f_{\rho}^{\mathrm{e}}$ acting on the normal constituent is given by exactly the same formula (29) as before. Although it is irrelevant for the purposes of application of the action principle, it may be noted that instead of (34), the remainder current in the final divergence term of (48) will be given by the rather different formula

$$
\mathcal{R}_{\mathrm{I}}^{\sigma}=\frac{\hbar}{2} \varphi^{\Upsilon}\left(\delta n_{\Upsilon}^{\sigma}+\frac{1}{2} n_{\Upsilon}^{\sigma} g^{\mu \nu} \delta g_{\mu \nu}\right)+2 \Pi_{\nu} \xi_{\mathrm{e}}^{[\sigma} u^{\nu]}+\frac{1}{4 \pi} F^{\nu \sigma} \delta A_{\nu} .
$$

More to the point for practical purposes, the stress energy momentum density tensor $T^{\mu \nu}$ in (48) will be given by the same formulae (31), (32), (33) as before, apart from the fact that in the new version the relevant generalised pressure function will be given by

$$
\Psi_{\mathrm{M}}=\Lambda_{\mathrm{M}}-\frac{\hbar}{2} n_{\mathrm{n}}^{\sigma} \nabla_{\sigma} \varphi^{\mathrm{n}}+n_{\mathrm{p}}^{\sigma}\left(e A_{\sigma}-\frac{\hbar}{2} \nabla_{\sigma} \varphi^{\mathrm{p}}\right)-n_{\mathrm{e}}^{\sigma} \mu_{\sigma}^{\mathrm{e}}+s \Theta,
$$

which can be seen to be equivalent to the original version, as given by (32), when the superconducting superfluid field equations (43) are satisfied, as is necessary in this modified formulation for invariance of the action with respect to arbitrary infinitesimal variations of $n_{\mathrm{n}}^{\rho}$ and $n_{\mathrm{p}}^{\rho}$.

The essential difference between a "normal" and a "super" current is not just that the latter is irrotational so that its momentum covector is locally proportional to the gradient of a scalar field, but more particularly that this scalar field will be globally definable as a periodic phase angle, so that the corresponding circulation integral will be quantised. Specifically, according to (44), the relevant neutron and proton circulation integrals for a given circuit will be given in the present case by

$$
\oint \pi_{\rho}^{r} d x^{\rho}=\pi \hbar \nu^{r}
$$

for a pair of integral proton and neutron phase winding numbers respectively given by $\nu^{\mathrm{n}}$ and $\nu^{\mathrm{p}}$. These numbers will remain constant as the circuit is continuously displaced, except when it crosses what, on a mesoscopic scale will be describable as a string like singularity, but what on a microscopic scale will be a vortex type defect whose core consists of a region in which the superfluidity property must break down. It is expected that, in most of the relevant neutron star layers, conditions will be such as to ensure that such vortex defects will generally be of elementary type, meaning that they will either be simple neutron flow vortices, as characterised, for an appropriate choice of orientation, by winding numbers $\nu^{\mathrm{n}}=1, \nu^{\mathrm{p}}=0$, or simple proton vortices, as similarly characterised by $\nu^{\mathrm{n}}=0, \nu^{\mathrm{p}}=1$. In order to obtain a description that is applicable on a macroscopic scale, it will evidently be necessary to average over a large number of such (elementary, or in more exotic circumstances, higher order) vortices. Following the example of our treatment of the single constituent case[3], the purpose of the next two sections is to develop and show how to apply the kind of formalism needed to achieve this. 


\section{Macroscopic allowance for vorticity fibration}

The standard (Taub type) flow line variational procedure illustrated by the category of models set up in Section 2 has the advantage of being extremely versatile: for example it can be generalised for application to simple elastic solids 13, as well as to conducting solids [16], and it has been used most recently for setting up the "transfusive" twoconstituent model[1] that has been designed to provide a crude but globally applicable description of a neutron star as a whole. However for the purpose of dealing with the macroscopic effects of vorticity quantisation, we have found [3] that it is more convenient to start off on the basis of a very different kind of variational procedure.

The standard procedure, as used in Section 2, for the treatment of any current, $n^{\sigma}$ say, is based on a projection of the four dimensional space-time background onto a three-dimensional material base space, whose local coordinates can then be pulled to specify corresponding comoving space coordinates on the space-time background: the dual three-form, ${ }^{*} n_{\nu \rho \sigma}$, is obtained as the pullback of a prescribed volume measure on the base space. In the alternative variation procedure, which has been shown to lead ultimately to the same equations of motion, both for a simple perfect fluid[2] and for a perfectly conducting two-constituent model [4], an analogous projection construction is used to specify not the current three-form ${ }^{*} n_{\nu \rho \sigma}$ but the corresponding vorticity two form $w_{\rho \sigma}$, which is to be obtained as the pullback of a prescribed area measure on a two-dimensional base space. In terms of suitably chosen local coordinates $\chi^{1}, \chi^{2}$ on the vorticity base manifold, the corresponding pair of scalar fields induced on the four dimensional spacetime background will specify the vorticity according to the formula

$w_{\rho \sigma}=2 \chi_{,[\rho}^{1} \chi_{, \sigma]}^{2}$. Such a prescription automatically ensures that the vorticity two-form will satisfy both the algebraic degeneracy condition

$$
w_{[\mu \nu} w_{\rho \sigma]}=0
$$

and the closure condition

$$
\nabla_{[\nu} w_{\rho \sigma]}=0
$$

For the purposes of the variational principle it is to be postulated that the projection of the four dimensional space-time background onto the two dimensional vorticity base space should be freely variable, which is equivalent to the postulate that $\chi^{1}$ and $\chi^{2}$ should be considered as freely variable local fields on spacetime. As for the three dimensional projection used for the direct specification of a current, so also for the two dimensional projection used for the specification of a vorticity form, it is convenient to represent an infinitesimal variation via an infinitesimal displacement vector field, $\xi^{\rho}$ say, in terms of which the corresponding base coordinate variations will be given by $\delta \chi^{1}=\xi^{\rho} \chi_{, \rho}^{1}, \delta \chi^{2}=\xi^{\rho} \chi_{, \rho}^{2}$. As in the case of currents, the use of such displacement vector fields to specify the corresponding "convective" variations enables us to employ a formalism that is manifestly invariant with respect to changes of the base coordinates, whose explicit use can thereby be avoided in the work that follows. It can easily be seen that a base coordinate independent expression for the infinitesimal variation induced 
by the displacement field $\xi^{\rho}$ will be given by the formula

$$
\delta w_{\mu \nu}=-2 \nabla_{[\mu}\left(w_{\nu] \rho} \xi^{\rho}\right) .
$$

This is evidently interpretable, taking account of the closure property (52), as the condition that the infinitesimal variation of $w_{\mu \nu}$ should be given simply by its Lie derivative with respect to the $\xi^{\mu}$.

The foregoing procedure makes it possible to treat vorticity as an independent field in its own right. However in such an approach the essential identity of the vorticity two form as the exterior derivative of a corresponding momentum one-form must be ultimately be established as a dynamical equation, instead of being imposed in advance as a defining property in the manner used in the preceding sections. In order to obtain the required exterior differential relation between momentum and vorticity in a variational treatment, it has been found convenient [2, 4, 3] to replace the standard three-dimensional base projection procedure used (as described above) for the specification of the relevant current, $n^{\rho}$ say, by an alternative Kalb-Ramond type procedure whereby the current is specified in terms of a freely variable gauge two-form, i.e. an antisymmetric tensor field $B_{\mu \nu}=-B_{\nu \mu}$ in terms of which the relevant current three-form is given by

$$
{ }^{*} n_{\mu \nu \rho}=3 \nabla_{[\mu} B_{\nu \rho]} .
$$

By the Poincaré lemma and its inverse, the existence of such a gauge potential is a sufficient and locally necessary condition for the closure property

$$
\nabla_{[\mu}^{*} n_{\nu \rho \sigma]}=0
$$

which is equivalent to the conservation law

$$
\nabla_{\rho} n^{\rho}=0
$$

The equivalent statement in dual terminology is that this conservation law (57) is the necessary and sufficient condition for the local existence of a bivector field $b^{\mu \nu}=-b^{\nu \mu}$ such that

$$
n^{\rho}=\nabla_{\sigma} b^{\rho \sigma}
$$

where $b^{\mu \nu}$ is the dual of a corresponding Kalb-Ramond form in terms of which it will be given by

$$
b^{\mu \nu}=\frac{1}{2} \varepsilon^{\mu \nu \rho \sigma} B_{\rho \sigma} .
$$

It is to be noted that a Kalb-Ramond representation of the form (55), or equivalently (58), can not be used in cases such that of the globally applicable "transfusive" model [1] in which the relevant currents are not separately conserved. It is also to be remarked that (assuming it is admissible) such a representation will evidently not be unique: it is subject to gauge transformations specifiable in terms of an arbitrary one-form $\alpha_{\nu}$ by

$$
B_{\mu \nu} \mapsto B_{\mu \nu}+2 \nabla_{[\mu} \alpha_{\nu]}
$$


In the particular case of the three constituent models with which the present discussion is concerned, the considerations presented above lead us to introduce an isotopic doublet of free gauge bivector fields $b_{\mathrm{n}}^{\rho \sigma}$ and $b_{\mathrm{p}}^{\rho \sigma}$ in terms of which the neutron and proton currents will be given by the combined expression

$$
n_{\Upsilon}^{\rho}=\nabla_{\sigma} b_{\Upsilon}^{\rho \sigma}
$$

where, as in the previous section, it is to be understood that the isotopic index runs over the values $\Upsilon=\mathrm{n}$ and $\Upsilon=\mathrm{p}$. However since the problem of vorticity quantisation does not arise for the normal constituent we shall continue to treat it as before, meaning that $n_{\mathrm{e}}^{\rho}$ will be supposed to be defined, via a projection, as the dual of the pullback of the measure on a three dimensional base space. Treating the gauge fields $b_{\mathrm{n}}^{\rho \sigma}$ and $b_{\mathrm{p}}^{\rho \sigma}$ as freely variable, but treating the associated vorticities $w_{\rho \sigma}^{\mathrm{n}}$ and $w_{\rho \sigma}^{\mathrm{p}}$, via independent projections, as pullbacks of the measure on a two dimensional base space in the manner described above, in a new Lagrangian obtained from (9) by a replacement

$$
\mathcal{L} \mapsto \mathcal{L}_{\mathbb{I I}}
$$

where (summing as usual over the isotopic index $\Upsilon$ )

$$
\mathcal{L}_{\mathbb{I I}}=\mathcal{L}+\frac{1}{2} b_{\Upsilon}^{\sigma \rho} w_{\rho \sigma}^{\Upsilon},
$$

it can be verified that one obtains a variational formulation leading back to field equations precisely equivalent to those obtained by the original procedure in Section 2 .

The advantage of the new procedure is that, following the example of our singleconstituent prototype 3], it can easily be generalised so as to provide a macroscopic description of the effects (whose analysis in a Newtonian framework was pioneered by Bekarevich and Khalatnikov 23, 24]) to be expected as a consequence of vortex quantisation, by replacing the original formula (10) for the gauge independent action contribution $\Lambda$ in the new Lagrangian

$$
\mathcal{L}_{\mathbb{I I}}=\Lambda+j^{\rho} A_{\rho}+\frac{1}{2} b_{\Upsilon}^{\sigma \rho} w_{\rho \sigma}^{\Upsilon},
$$

by a more general formula according to which $\Lambda$ is given as an algebraic function not just of the (gauge independent) fields $n_{\mathrm{n}}^{\rho}, n_{\mathrm{p}}^{\rho}, n_{\mathrm{e}}^{\rho}, s, F_{\mu \nu}$, and of course the metric $g_{\mu \nu}$, as before, but also on the (similarly gauge independent) doublet of vorticity two-forms, $w_{\rho \sigma}^{\mathrm{n}}$ and $w_{\rho \sigma}^{\mathrm{p}}$. This means that its most general infinitesimal variation will be given by an expression of the form

$$
\delta \Lambda=\mu_{\rho}^{\Upsilon} \delta n_{\Upsilon}^{\rho}+\frac{1}{2} \lambda_{\Upsilon}^{\sigma \rho} \delta w_{\rho \sigma}^{\Upsilon}+\mu_{\rho}^{\mathrm{e}} \delta n_{\mathrm{e}}^{\rho}-\Theta \delta s+\frac{1}{8 \pi} \mathcal{H}^{\sigma \rho} \delta F_{\rho \sigma}+\frac{\partial \Lambda}{\partial g_{\rho \sigma}} \delta g_{\rho \sigma},
$$

where, as in (5), the coefficients of the metric variations are not independent of the others but by the relevant generalisation of the Noether identity (6) must satisfy

$$
\frac{\partial \Lambda}{\partial g_{\rho \sigma}}=\frac{\partial \Lambda}{\partial g_{\sigma \rho}}=\frac{1}{2} \mu_{\nu}^{\mathrm{x}} n_{\mathrm{x}}^{\rho} g^{\nu \sigma}+\frac{1}{2} \lambda_{\Upsilon}^{\nu \rho} w_{\nu}^{\gamma \sigma}+\frac{1}{16 \pi} \mathcal{H}^{\nu \rho} F_{\nu}{ }^{\sigma}
$$


The new doublet of bivectorial coefficients $\lambda_{r}^{\sigma \rho}=-\lambda_{r}^{\rho \sigma}$ in this expansion characterises the macroscopic anisotropy arising respectively from the concentration of energy and tension in mesoscopic vortices of the neutron and proton superfluids as a consequence of their vorticity quantisation conditions, in the manner discussed in our previous work [3] on the single constituent model. These new four dimensional bivectorial coefficients replace the three dimensional (space) vectorial coefficients introduced for a similar purpose in a more restricted Newtonian framework by Bekarevich and Khalatnikov 23. As before, the covectorial coefficients $\mu_{\rho}^{\mathrm{n}}, \mu_{\rho}^{\mathrm{p}}$, and $\mu_{\rho}^{\mathrm{e}}$ will be interpretable respectively as the mean momentum covectors per particle of the neutrons, the protons, and the electrons, and $\Theta$ will be interpretable as the temperature of the "normal constituent". Finally the bivectorial coefficient $\mathcal{H}^{\rho \sigma}=-\mathcal{H}^{\sigma \rho}$ will be interpretable as an electromagnetic displacement tensor, in terms of which the total electromagnetic field tensor (11) will be given by an expression of the form

$$
F^{\rho \sigma}=\mathcal{H}^{\rho \sigma}+4 \pi \mathcal{M}^{\rho \sigma}
$$

in which $\mathcal{M}^{\rho \sigma}$ is what can be interpreted as the magnetic polarisation tensor. In the application that we are considering, the - typically dominant - polarisation contribution $4 \pi \mathcal{M}^{\rho \sigma}$ is to be thought of as representing the part of the magnetic field confined in the vortices, while the - typically much smaller - remainder $\mathcal{H}^{\rho \sigma}$ represents the average contribution from the external field in between the vortices, which can be expected to vanish by the "Meissner effect" in strictly static configurations, but which can be expected to acquire a non zero value due to the "London effect" in rotating configurations.

The analogue of (26), as the macroscopic replacement of (48), for an infinitesimal variation according to the foregoing rules - with the neutron and proton vorticity congruences convected by displacement vector fields $\xi_{\mathrm{n}}^{\rho}$ and $\xi_{\mathrm{p}}^{\rho}$, and with the flow lines of the "normal" constituent convected by a displacement vector field $\xi_{\mathrm{e}}^{\rho}$ as before - will be a "diamond" variation (i.e. one that includes allowance for the effect on the background spacetime measure) expressible in the standard form

$$
\diamond \mathcal{L}_{\mathbb{I}}=\left(\nabla_{\rho} \pi_{\sigma}^{\Upsilon}-\frac{1}{2} w_{\rho \sigma}^{\Upsilon}\right) \delta b_{\Upsilon}^{\rho \sigma}+f_{\rho}^{\mathrm{x}} \xi_{\mathrm{x}}^{\rho}+\left(j^{\rho}-\frac{1}{4 \pi} \nabla_{\sigma} \mathcal{H}^{\rho \sigma}\right) \delta A_{\rho}+\frac{1}{2} T^{\mu \nu} \delta g_{\mu \nu}+\nabla_{\sigma} \mathcal{R}_{\mathbb{I}}{ }^{\sigma},
$$

in which the force densities $f_{\rho}^{\mathrm{x}}$ appearing as coefficients of the three independent displacement displacement vector fields $\xi_{\mathrm{x}}^{\rho}$ will be given for the isotopic doublet values $\mathrm{X}=\mathrm{n}$ and $\mathrm{X}=\mathrm{p}$ by

$$
f_{\rho}^{\mathrm{n}}=\left(n_{\mathrm{n}}^{\sigma}+\nabla_{\nu} \lambda_{\mathrm{n}}^{\sigma \nu}\right) w_{\sigma \rho}^{\mathrm{n}}, \quad f_{\rho}^{\mathrm{p}}=\left(n_{\mathrm{p}}^{\sigma}+\nabla_{\nu} \lambda_{\mathrm{p}}^{\sigma \nu}\right) w_{\sigma \rho}^{\mathrm{p}},
$$

while the third force density $f_{\rho}^{\mathrm{e}}$ acting on the "normal" constituent will still be given by the same formula (29) as before.

Although it is of no relevance for the application of the variation principle, it can be noted for the record that the current appearing in the final divergence term of (68) will be given by

$$
\mathcal{R}_{\mathbb{I}}^{\sigma}=\pi_{\rho}^{\Upsilon} \delta b_{\Upsilon}^{\rho \sigma}-\left(b_{\Upsilon}^{\rho \sigma}+\lambda_{\Upsilon}^{\rho \sigma}\right) w_{\rho \nu}^{\Upsilon} \xi_{\Upsilon}^{\nu}+\frac{1}{2} \pi_{\rho}^{\Upsilon} b_{\Upsilon}^{\rho \sigma} g^{\mu \nu} \delta g_{\mu \nu}+2 \Pi_{\nu} \xi_{\mathrm{e}}^{[\sigma} u^{\nu]}+\frac{1}{4 \pi} H^{\nu \sigma} \delta A_{\nu} .
$$


An entity of much greater practical interest is the corresponding stress momentum energy density tensor, which can be seen to be given by

$$
T_{\sigma}{ }^{\rho}=n_{\mathrm{x}}^{\rho} \mu_{\sigma}^{\mathrm{x}}+s \Theta u^{\rho} u_{\sigma}+\lambda_{\mathrm{n}}^{\nu \rho} w_{\nu \sigma}^{\mathrm{n}}+\lambda_{\mathrm{p}}^{\nu \rho} w_{\nu \sigma}^{\mathrm{p}}+\frac{1}{8 \pi} \mathcal{H}^{\nu \rho} F_{\nu \sigma}+\Psi g_{\sigma}^{\rho},
$$

where the new generalised pressure function is given by

$$
\Psi=\Lambda-n_{\mathrm{x}}^{\nu} \mu_{\nu}^{\mathrm{x}}+s \Theta+b_{\Upsilon}^{\rho \sigma}\left(\nabla_{\rho} \pi_{\sigma}^{\Upsilon}-\frac{1}{2} w_{\rho \sigma}^{\Upsilon}\right)
$$

The last term in (72) will evidently drop out when we impose the condition of invariance with respect to infinitesimal variations of the bivectorial gauge potentials $b_{\mathrm{n}}^{\rho \sigma}$ and $b_{\mathrm{p}}^{\rho \sigma}$ is imposed, a requirement which can be seen from (68) to give field equations of the form

$$
w_{\rho \sigma}^{\mathrm{n}}=2 \nabla_{[\rho} \pi_{\sigma]}^{\mathrm{n}}=2 \nabla_{[\rho} \mu_{\sigma]}^{\mathrm{n}}, \quad w_{\rho \sigma}^{\mathrm{p}}=2 \nabla_{[\rho} \pi_{\sigma]}^{\mathrm{p}}=2 \nabla_{[\rho} \mu_{\sigma]}^{\mathrm{p}}+e F_{\rho \sigma} .
$$

These expressions are evidently equivalent to what in the previous formulation were merely the definitions of the vorticities as given by the general specification (15).

The remaining field equations obtained from (68) will consist of an electromagnetic source equation having the form

$$
\nabla_{\sigma} \mathcal{H}^{\rho \sigma}=4 \pi j^{\rho}
$$

together with the condition that the force density coefficients should all vanish. For the normal constituent, the corresponding condition

$$
f_{\rho}^{\mathrm{e}}=0
$$

will take the same form (42) as before, but it can be seen from (69) that the other two force balance conditions,

$$
f_{\rho}^{\Upsilon}=0,
$$

will involve new terms. In particular, the neutron force balance condition can be seen from (69) to be expressible as

$$
2 n_{\mathrm{n}}^{\sigma} \nabla_{[\sigma} \mu_{\rho]}^{\mathrm{n}}+w_{\sigma \rho}^{\mathrm{n}} \nabla_{\nu} \lambda_{\mathrm{n}}^{\sigma \nu}=0,
$$

in which the first term is interpretable as the negative of the Joukowski force density due to the "Magnus effect" acting on the neutron vortices, while the last term (which was absent in the mesoscopic description) represents the extra force density on the fluid due to the effect of the tension of the vortices. The proton force balance equation can be similarly be seen to be expressible in the form

$$
2 n_{\mathrm{p}}^{\sigma} \nabla_{[\sigma} \mu_{\rho]}^{\mathrm{p}}+e n_{\mathrm{p}}^{\sigma} F_{\sigma \rho}+w_{\sigma \rho}^{\mathrm{p}} \nabla_{\nu} \lambda_{\mathrm{p}}^{\sigma \nu}=0,
$$

in which the first term is the negative of the Joukowski force density that acts on the proton vortices, the middle term is the Lorentz force density representing the effect of the magnetic field on the passing protons, and the final term again represents the force density due to the effect of the tension of the vortices. 


\section{Entrainment in partially separated models.}

In order to be utilisable for particular applications, the characterisation of the mesoscopic and macroscopic models described in the preceding sections needs to be completed by the designation of an appropriate "equation of state" giving the specific form of the mesoscopic $\Lambda_{\mathrm{M}}$ and the macroscopic $\Lambda$ as algebraic functions of the relevant currents and also, for the latter, of the relevant vorticities as well as the electromagnetic field. Accurately realistic "equations of state" can only be obtained by detailed microscopic analysis, and even if available would be likely to be too complicated to be convenient in practice. Since use of a conservative model involving only three independent constituents is already a simplification, it is reasonable to employ a correspondingly simplified "equation of state".

A very convenient kind of simplification, that is implicitly or explicitly employed in most of the preceding work in a Newtonian framework [6, 7], and that will be adopted for the work of the present section, is to suppose that the action contribution (and hence the corresponding stress momentum energy contribution) of the "superfluid" or "quantised" part is separate from that of the "normal" or strictly "classical" part, meaning that at the level of the "mesoscopic" description the relevant "matter" contribution $\Lambda_{\mathrm{M}}$ is taken to be decomposable as a sum in the form

$$
\Lambda_{\mathrm{M}}=\Lambda_{\mathrm{Q}}+\Lambda_{\mathrm{C}}
$$

where, in the three constituent models with which we are concerned here, the "quantum" part depends only on the neutron and the proton currents, while the "classical" part of the Lagrangian density depends only on the comoving electron and entropy currents, so that the variation (7) decomposes as the sum of two separate contributions

$$
\delta \Lambda_{\mathrm{Q}}=\mu_{\rho}^{\Upsilon} \delta n_{\Upsilon}^{\rho}+\frac{1}{2} \mu_{\nu}^{\Upsilon} n_{\Upsilon}^{\rho} g^{\nu \sigma} \delta g_{\rho \sigma}
$$

and

$$
\delta \Lambda_{\mathrm{C}}=\mu_{\rho}^{\mathrm{e}} \delta n_{\mathrm{e}}^{\rho}-\Theta \delta s+\frac{1}{2} \mu_{\nu}^{\mathrm{e}} n_{\mathrm{e}}^{\rho} g^{\nu \sigma} \delta g_{\rho \sigma} .
$$

It seems plausible that such a simplification should be fairly satisfactory for the low temperature limit, $\Theta \rightarrow 0$, thus providing an adequate approximation for many purposes in neutron star theory. However as was clearly understood by Landau when he obtained the equation of state for the "cool" limit of his original two constituent model 25] (of which an elegant relativistic analogue has recently been provided by the present authors 26]) there will inevitably be significant coupling between the superfluid and "normal" constituents at higher temperatures, for which the decomposition (79) would therefore cease to be valid.

The simplest conceivable models would be based on not just partial but complete separation, which would mean that the contribution $\Lambda_{\mathrm{Q}}$ in (79) would itself split up as the sum of two distinct terms, $\Lambda_{\mathrm{n}}$ and $\Lambda_{\mathrm{p}}$ say, of which the first depends only on the magnitude of the neutron current vector, and the second only on that of the 
proton current vector. However ever since the earliest pioneering work by Andreev and Bashkin 19 it has been generally recognised that (even in the zero temperature limit) such an extreme simplification will be unrealistic. This means that the form of $\Lambda_{\mathrm{Q}}$ will be such that the proton and neutron momenta will be given in terms of the corresponding currents by a (necessarily symmetric) matrix relation of the form

$$
\mu_{\rho}^{\Upsilon}=g_{\rho \sigma} \mathcal{K}^{\Upsilon \Phi} n_{\Phi}^{\sigma}
$$

in which, as well as the diagonal terms $\mathcal{K}^{\mathrm{nn}}$ and $\mathcal{K}^{\mathrm{pp}}$ that would be present even in the oversimplified fully separated case, there will also be a cross term $\mathcal{K}^{\mathrm{np}}=\mathcal{K}^{\mathrm{np}}$. The presence of such a term implies that the respective directions of the neutron and proton momenta will deviate from that of the corresponding currents, in a manner that is familiar in the well known example of the Landau type two constituent model at non-zero temperature. Such a (non-dissipative) deviation effect is usually referred to as entrainment. (It is however to be noted that there is an alternative but regrettable usage whereby some authors call it "drag", which is misleading, since the standard use of the latter term is for dissipative forces opposing relative movement.) The (symmetric) 2 by 2 matrix relation (82) can be rewritten in inverted form as

$$
n_{\Upsilon}^{\rho}=g^{\rho \sigma} \mathcal{K}_{\Upsilon \Phi} \mu_{\sigma}^{\Phi}
$$

using the usual convention that (just as $g^{\rho \sigma}$ denotes the components of the matrix inverse of the metric tensor $g_{\rho \sigma}$ ) the matrix with (lowered) components $\mathcal{K}_{{ }_{\Upsilon \Phi}}$ is the inverse of the original matrix with components $\mathcal{K}^{\Upsilon \Phi}$.

For the purpose of comparison with our treatment[3] of the single constituent case, for which it was found convenient to work with a "dilatonic amplitude", $\Phi$ defined as the square root of the ratio of the particle number density to the effective mass, it is of interest to introduce analogous amplitudes $\Phi_{\mathrm{n}}$ and $\Phi_{\mathrm{p}}$ for the protons and neutrons respectively in such a way as to allow (83) to be rewritten more explicitly as

$$
n_{\mathrm{n} \sigma}=\Phi_{\mathrm{n}}^{2} \mu_{\sigma}^{\mathrm{n}}+\mathcal{K}_{\mathrm{np}}\left(\mu_{\sigma}^{\mathrm{p}}-\mu_{\sigma}^{\mathrm{n}}\right), \quad n_{\mathrm{p} \sigma}=\Phi_{\mathrm{p}}^{2} \mu_{\sigma}^{\mathrm{p}}+\mathcal{K}_{\mathrm{np}}\left(\mu_{\sigma}^{\mathrm{n}}-\mu_{\sigma}^{\mathrm{p}}\right)
$$

which evidently requires the specifications

$$
\Phi_{\mathrm{n}}^{2}=\mathcal{K}_{\mathrm{nn}}+\mathcal{K}_{\mathrm{np}}, \quad \Phi_{\mathrm{p}}^{2}=\mathcal{K}_{\mathrm{pp}}+\mathcal{K}_{\mathrm{np}}
$$

In order to relate the formula to $(82)$ to the expressions traditionally used in the relevant literature as previously developed in a Newtonian framework [19, 28, 7], it is convenient to introduce what from a relativistic point of view is a rather artificial concept, namely that of the "rest mass", $m$ say per baryon, which can indifferently be taken to be the mass of a free neutron, the (more precisely definable) mass of a free proton, or as a compromise between the two, the mass of an ordinary free hydrogen atom in its ground state, or even the standard atomic unit as conventionally defined in terms of oxygen. The reason why it does not matter which of these one uses is that this "rest mass" is needed merely as a unit for calibration, and in any case by the standards 
of accuracy of the present treatment all these various alternative definitions give are virtually the same result. However the calibration mass $m$ is chosen, one can use it to define corresponding conserved "mass currents" $\rho_{\mathrm{n}}^{\nu}$ and $\rho_{\mathrm{p}}^{\nu}$ for the protons and neutrons by the formula

$$
\rho_{\Upsilon}^{\nu}=m n_{\Upsilon}^{\nu}
$$

Another popular concept in the Newtonian context, despite the fact that its calibration is subject to a similar degree of arbitrariness, is that of what is commonly but misleadingly referred to as a superfluid "velocity". One can define such so called "velocities" $v^{\mathrm{n} \nu}$ and $v^{\mathrm{p} \nu}$ for the neutrons and the protons in terms of the chosen calibration mass $m$ (as specified by one or other of the various conventions mentioned above for defining the "baryon rest mass") by setting

$$
\mu_{\nu}^{r}=m v_{\nu}^{r}
$$

which means that $v_{\nu}^{\mathrm{n}}$ and $v_{\nu}^{\mathrm{p}}$ can be read out just as correspondingly rescaled momentum variables. It is to be observed that, no matter how the constant mass scale factor $m$ is chosen, $v^{\mathrm{n} \nu}$ and $v^{\mathrm{p} \nu}$ can not be made to be "velocity" 4 -vectors in the strict sense because their magnitudes will in general be variable, so that they can not be made to be unit vectors, except in such trivial special cases as that of a spatially uniform stationary state. In terms of these so called "velocities", which are actually just rescaled momentum variables, (83) can be rewritten in the form

$$
\rho_{\Upsilon}^{\rho}=g^{\rho \sigma} \rho_{\Upsilon \Phi} v_{\sigma}^{\Phi}
$$

where the coefficients $\rho_{\Upsilon \Phi}$ are components of a "density matrix" of the traditional but unsatisfactorily mass scale dependent kind, which will be given in terms of the more precisely defined matrix components $\mathcal{K}_{\Upsilon \Phi}$ introduced above by

$$
\rho_{\Upsilon \Phi}=m^{2} \mathcal{K}_{\Upsilon \Phi}
$$

Writing this out using the definitions

$$
\rho_{\mathrm{n}}=\left(m \Phi_{\mathrm{n}}\right)^{2}=\rho_{\mathrm{nn}}+\rho_{\mathrm{np}}, \quad \rho_{\mathrm{p}}=\left(m \Phi_{\mathrm{p}}\right)^{2}=\rho_{\mathrm{pp}}+\rho_{\mathrm{np}},
$$

it can can be be seen that when translated into terms of the pseudo-velocities, the expansions (84) will take the kind of form that is familiar in the Newtonian literature:

$$
\rho_{\mathrm{n} \sigma}=\rho_{\mathrm{n}} v_{\sigma}^{\mathrm{n}}+\rho_{\mathrm{np}}\left(v_{\sigma}^{\mathrm{p}}-v_{\sigma}^{\mathrm{n}}\right), \quad \rho_{\mathrm{p} \sigma}=\rho_{\mathrm{p}} v_{\sigma}^{\mathrm{p}}+\rho_{\mathrm{np}}\left(v_{\sigma}^{\mathrm{n}}-v_{\sigma}^{\mathrm{p}}\right) .
$$

\section{Polarisation in partially separated models.}

For the purpose of extending the kind of "mesoscopic" model considered in the previous section to a "macroscopic" model representing the effect of averaging over a congruence of vortex lines in the manner described in Section 1 , it is useful to start by using the 
relations obtained in Section 5 to obtain rough estimates of the total electromagnetic flux,

$$
\Phi=\oint A_{\sigma} d x^{\sigma}
$$

that is to be expected through a circuit at sufficiently large distance round a vortex defect characterised, according to (51) by neutron and proton and winding numbers $\nu^{\mathrm{n}}$ and $\nu^{\mathrm{p}}$. It can be seen, by working out (83) for the particular case of the proton current $n_{\mathrm{p}}^{\sigma}$ that the total value of the electromagnetic gauge field will be expressible in the form

$$
A_{\sigma}=\frac{1}{e}\left(\pi_{\sigma}^{\mathrm{p}}+\alpha_{\mathrm{n}}^{\mathrm{p}} \pi_{\sigma}^{\mathrm{n}}\right)+\Delta_{\sigma}
$$

where the factor $\alpha_{\mathrm{n}}^{\mathrm{p}}$ is a quantity that would be zero if there were no "entrainment" but that will in general be given by

$$
\alpha_{\mathrm{n}}^{\mathrm{p}}=\frac{\mathcal{K}_{\mathrm{pn}}}{\mathcal{K}_{\mathrm{pp}}}=\frac{\rho_{\mathrm{pn}}}{\rho_{\mathrm{pp}}},
$$

while the final remainder term will be given by

$$
\Delta^{\sigma}=-\frac{1}{e \mathcal{K}_{\mathrm{pp}}} n_{\mathrm{p}}{ }^{\sigma}=-\frac{m}{e \rho_{\mathrm{pp}}} \rho_{\mathrm{p}}^{\sigma} .
$$

Assuming that the coefficients $\mathcal{K}_{{ }_{\Upsilon \Phi}}$ tend approximately towards uniform values at large distance, the corresponding flux $\Phi$ will tend approximately towards a limit given in terms of these values by

$$
\Phi \simeq \nu^{\Upsilon} \Phi_{\Upsilon}+\Phi_{\Delta}
$$

where, by (51), the proton vortex flux contribution will be given simply by

$$
\Phi_{\mathrm{p}}=\frac{\pi \hbar}{e}
$$

which is just the usual flux quantisation unit associated with the relevant total charge $2 e$ of the Cooper type proton pairs, while the neutron vortex flux contribution would vanish if there were no "entrainment" but will in general be given by the formula

$$
\Phi_{\mathrm{n}}=\frac{\pi \hbar}{e} \alpha_{\mathrm{n}}^{\mathrm{p}}
$$

where $\alpha_{\mathrm{n}}^{\mathrm{p}}$ is the relevant "entrainment factor". The formula (98), with $\alpha_{\mathrm{n}}^{\mathrm{p}}$ given by the final expression in (94), is already familiar from the corresponding analysis in a Newtonian framework 27, 28]. If the "Meissner effect" were fully effective the residual flux term would tend to zero, but as was originally realised by London in the context of ordinary metallic superconductivity this can be expected only in a strictly static background, whereas more generally even in a stationary case provided the background is rotating, there will be a residual magnetic field even at large distances from a vortex, 
and hence a corresponding "London" flux contribution, which will be given in the present case by

$$
\Phi_{\Delta}=-\frac{1}{e \mathcal{K}_{\mathrm{pp}}} \oint n_{\mathrm{p}}^{\rho} g_{\rho \sigma} d x^{\sigma}
$$

Under reasonably stable conditions one would expect that energy would be locally minimised by avoidance of the field build up that would result from persistent deviation of the proton current contribution $n_{\mathrm{p}}^{\sigma}$ from the canceling background current contribution $n_{\mathrm{e}}^{\sigma}$ provided by the "normal" electrons, i.e. one would expect to have

$$
n_{\mathrm{p}}^{\sigma} \approx n_{\mathrm{e}} u^{\sigma}
$$

where $u^{\mu}$ is the unit 4-velocity vector of the "normal" background, as introduced above. Under such circumstances, the "London" flux contribution (99) can be expected to be given in terms of the asymptotic values of the proton number density $n_{\mathrm{p}}$ or the corresponding conventionally normalised proton mass density as defined by

$$
n_{\mathrm{p}}=\left(-n_{\mathrm{p}}^{\sigma} n_{\mathrm{p} \sigma}\right)^{1 / 2}, \quad \rho_{\mathrm{p}}=m n_{\mathrm{p}},
$$

by an estimate of the form

$$
\Phi_{\Delta} \approx-\frac{n_{\mathrm{p}}}{e \mathcal{K}_{\mathrm{pp}}} \oint u_{\sigma} d x^{\sigma}=-\frac{m \rho_{\mathrm{p}}}{e \rho_{\mathrm{pp}}} \oint u_{\sigma} d x^{\sigma}
$$

However that may be, the "London" flux contribution $\Phi_{\Delta}$ through a circuit round a plane surface element of area $\mathcal{S}$ can be attributed to an average orthogonally oriented magnetic field contribution $\langle H\rangle$ given by

$$
\langle H\rangle=\frac{\Phi_{\Delta}}{\mathcal{S}} .
$$

In addition to this external London contribution, the corresponding total average magnetic field, as defined by

$$
\langle B\rangle=\frac{\Phi}{\mathcal{S}}
$$

will contain an internal contribution from the part of the flux that is confined within the neighbourhood of the vortex. According to (96), this internal contribution will be expressible by

$$
\langle B\rangle-\langle H\rangle=\frac{1}{\pi \hbar} \Phi_{\Upsilon}\left\langle w^{\Upsilon}\right\rangle,
$$

where the effective average neutron and proton vorticities $\left\langle w^{\mathrm{n}}\right\rangle$ and $\left\langle w^{\mathrm{p}}\right\rangle$ are given in terms of the corresponding quantised circulation integrals (51) by

$$
\left\langle w^{r}\right\rangle=\frac{1}{\mathcal{S}} \oint \pi_{\rho}^{r} d x^{\rho}
$$

The formula (105) provides what one needs for going over from the "mesoscopic" level of analysis we have been using so far in this section to a "macroscopic" level of 
analysis in terms of averages over many vortices. The average magnitude $\left\langle w^{\Upsilon}\right\rangle$ is interpretable as the relevant component (as determined by the choice of orientation of the circuit under consideration) of the kind of macroscopic vorticity tensor $w_{\mu \nu}^{r}$ that was considered in the preceding section. The total average magnitude $\langle B\rangle$ is analogously interpretable as the corresponding component of the macroscopic electromagnetic field tensor $F_{\rho \sigma}$, while the external "London" contribution $\langle H\rangle$ will be similarly interpretable as the corresponding component of the generalised displacement field tensor $\mathcal{H}_{\rho \sigma}$ that was introduced in the previous section. By comparison with (67), it can thus be seen that the difference (105) will be interpretable as the corresponding component of $4 \pi \mathcal{M}_{\rho \sigma}$, where $\mathcal{M}_{\rho \sigma}$ is the relevant polarisation tensor. It follows that this macroscopic polarisation tensor, representing the effect of the part of the magnetic field that is confined in the neighbourhood of the vortices, will be given in terms of the macroscopic proton and neutron vorticity tensors by

$$
4 \pi^{2} \hbar \mathcal{M}_{\rho \sigma}=\Phi_{\Upsilon} w_{\rho \sigma}^{r}
$$

It is important to point out that this result differs from the result obtained in the Newtonian limit by Mendell [7], who found the polarisation to be zero. However that conclusion was based on the inclusion of an extra magnetic interaction term but on the neglect of a kinetic contribution that, according to a more recent analysis [29], will actually cancel it out.

Working out the polarisation tensor explicitly in terms of the elementary proton and neutron vortex flux units (97) and (98) it can be seen that the Dirac-Planck constant $\hbar$ cancels out, leaving a relation of the form

$$
4 \pi e \mathcal{M}_{\rho \sigma}=w_{\rho \sigma}^{\mathrm{p}}+\alpha_{\mathrm{n}}^{\mathrm{p}} w_{\rho \sigma}^{\mathrm{n}},
$$

in which the last term on the right is entirely due to the "entrainment" effect, without which the neutron vorticity would not contribute to the magnetic polarisation. According to (67) the displacement field due to the "London effect" will be given by

$$
\mathcal{H}_{\mu \nu}=F_{\mu \nu}-\frac{1}{e}\left(w_{\mu \nu}^{\mathrm{p}}+\alpha_{\mathrm{n}}^{\mathrm{p}} w_{\mu \nu}^{\mathrm{n}}\right) .
$$

In terms of the "normal" background's acceleration vector $\dot{u}^{\nu}$ and rotation tensor $\Omega_{\mu \nu}$ (whose magnitude $\Omega=\left(\frac{1}{2} \Omega_{\mu \nu} \Omega^{\mu \nu}\right)^{1 / 2}$ is the local angular velocity) as defined by

$$
\nabla_{[\mu} u_{\nu]}=2 \Omega_{\mu \nu}-u_{[\mu} \dot{u}_{\nu]}, \quad \dot{u}^{\mu}=u^{\nu} \nabla_{\nu} u^{\mu},
$$

one would expect that in steady circumstances such that the estimate (102) is valid, the "London" field would be close to a value given by

$$
\mathcal{H}_{\mu \nu} \approx \frac{n_{\mathrm{p}}}{e \mathcal{K}_{\mathrm{pp}}}\left(u_{[\mu} \dot{u}_{\nu]}-2 \Omega_{\mu \nu}\right)=\frac{m \rho_{\mathrm{p}}}{e \rho_{\mathrm{pp}}}\left(u_{[\mu} \dot{u}_{\nu]}-2 \Omega_{\mu \nu}\right),
$$

in which it can be seen that the electric part is proportional to the acceleration while the magnetic part is proportional to the angular velocity. 
The "London field" (109) will be interpretable (whether or not the conditions for (111) are satisfied) as representing the contribution to the total macroscopic average electromagnetic field $F_{\mu \nu}$ from the magnetic field (if any) "outside" the vortices. Such a heuristic interpretation should not be taken too seriously because there will not be an absolutely clear cut boundary between the "inside" and the "outside" of a vortex. Nevertheless the distinction between the internal contribution described in terms of polarisation and the external contribution described as a "London" field will usually be fairly precise in practice, because the magnetic field can be expected to suffer an exponential decline with a lengthscale small compared to the intervortex separation.

In so far as there is some residual degree of arbitrariness in the distinction between the internal "polarisation" contribution and the external "London" contribution, the formula (109) can be considered as an exact defining condition. However this convention will only be consistent with the original definition of the displacement field via (65) in terms of partial differentiation with respect to the total field $F_{\mu \nu}$ if the Lagrangian density contribution $\Lambda$ has the right functional form. For mathematical consistency between (65) and (109) it is necessary and sufficient that $\Lambda$ should be decomposable in the form

$$
\Lambda=\Lambda_{\mathrm{MV}}+\Lambda_{\mathrm{F}},
$$

where the macroscopic contribution $\Lambda_{\mathrm{MV}}$ is required to be functionally independent of $F_{\mu \nu}$, though it is dependent now on the vorticities $w_{\rho \sigma}^{r}$ as well as on the currents, while the remaining contribution is required to have the specific form

$$
\Lambda_{\mathrm{F}}=\frac{1}{16 \pi} \mathcal{H}_{\rho \sigma} \mathcal{H}^{\sigma \rho}
$$

So long as the vortices are (as is expected to be the case in typical neutron star application) sufficiently far apart to justify the heuristic interpretation of $\mathcal{H}_{\mu \nu}$ as the intervortex contribution to the macroscopically averaged field $F_{\mu \nu}$, it is physically plausible that the action contribution of this "London" field should be given by substituting $\mathcal{H}_{\rho \sigma}$ for $F_{\mu \nu}$ in the standard Maxwellian formula (12). Since the formula (113) is precisely what results from such a substitution, the physical coherence of the description we have developed here is reassuringly confirmed.

In the context of the partial separation approximation on which the analysis of this section is based, there will be no loss of generality in decomposing the macroscopic Lagrangian density contribution $\Lambda_{\mathrm{MV}}$ in the form

$$
\Lambda_{\mathrm{MV}}=\Lambda_{\mathrm{M}}+\Lambda_{\mathrm{V}}
$$

where $\Lambda_{\mathrm{M}}$ is the function already used in the mesoscopic analysis, which in the present section is assumed to have the partially separated form (79), while $\Lambda_{\mathrm{V}}$ is an extra term which unlike $\Lambda_{\mathrm{M}}$ is algebraically dependent not just on the currents but also on the vorticity 2 -forms.

Before concluding, it is to be remarked that, for the purpose of comparison with previous work in Newtonian theory, it may be useful to introduce in our macroscopic model 
the notions of "velocities" and of "density matrix" that were defined in a mesoscopic context in Section 5. This will mean to replace the (in our opinion more natural) forms (77) and (78) of the respective neutron and proton equations of motion by alternative decompositions

$$
2 \rho_{\mathrm{n}} v^{\mathrm{n} \sigma} \nabla_{[\sigma} v_{\nu]}^{\mathrm{n}}=\rho_{\mathrm{np}}\left(v^{\mathrm{p} \sigma}-v^{\mathrm{n} \sigma}\right) m^{-1} w_{\nu \sigma}^{\mathrm{n}}+w_{\nu \sigma}^{\mathrm{n}} \nabla_{\rho} \lambda_{\mathrm{n}}^{\sigma \rho},
$$

and

$$
2 \rho_{\mathrm{p}} v^{\mathrm{p} \sigma} \nabla_{[\sigma} v_{\nu]}^{\mathrm{p}}=\rho_{\mathrm{np}}\left(v^{\mathrm{n} \sigma}-v^{\mathrm{p} \sigma}\right) m^{-1} w_{\nu \sigma}^{\mathrm{p}}-\frac{e}{m}\left(\rho_{\mathrm{pp}} v^{\mathrm{p} \sigma}+\rho_{\mathrm{np}} v^{\mathrm{n} \sigma}\right) F_{\sigma \nu}+w_{\nu \sigma}^{\mathrm{p}} \nabla_{\rho} \lambda_{\mathrm{p}}^{\sigma \rho},
$$

in each of which the first term on the right can be interpreted as an "entrainment force" density. It is to be observed that - due to the antisymmetry of the generalised vorticity tensors- such entrainment forces always act orthogonally to the relevant relative difference $v^{\mathrm{n} \sigma}-v^{\mathrm{p} \sigma}$, thereby respecting the strictly conservative character of the model. (The occasional use in the literature of the term "drag" instead of "entrainment" can be seen to be misleading, since a genuine drag force is intrinsically dissipative, and if present would not act orthogonally but on the contrary would be aligned with the relevant relative difference. In order to conform with standard aerodynamical terminology, the "entrainment" force should be described not as "drag" but rather as a kind of "lift".)

\section{Conclusions}

What remains for future work is the derivation of a suitable formula for the explicit form of the contribution $\Lambda_{\mathrm{MV}}$. Supposing that the vorticity independent mesoscopic contributions $\Lambda_{\mathrm{Q}}$ and $\Lambda_{\mathrm{C}}$ are known, the "entrainment ratio" needed for the specification via (113) of $\Lambda_{\mathrm{F}}$ will be given explicitly by (94), and one might hope that (as in the example of our preceding study of the single constituent case[3]) there might be a similarly simple and explicit formula for the corresponding form of the extra vorticity dependent term $\Lambda_{\mathrm{V}}$.

If the vortices were of purely "local" type, with not just their magnetic but also the associated circulating currents falling off exponentially in the exterior, then one would expect that $\Lambda_{\mathrm{V}}$ would just be a homogeneous linear function the magnitudes, $w^{\mathrm{n}}=\left(\frac{1}{2} w_{\mu}^{\mathrm{n} \nu} w_{\nu}^{\mathrm{n} \mu}\right)^{1 / 2}$ and $w^{\mathrm{p}}=\left(\frac{1}{2} w_{\mu}^{\mathrm{p} \nu} w_{\nu}^{\mathrm{p} \mu}\right)^{1 / 2}$ of the corresponding vorticity forms. In the absence of "entrainment" the elementary proton vortices would indeed be of this "local" type (like the electron vortices in ordinary "type II" metallic superconductors). However even in the single constituent case [3] the neutron vortices are of "global" type, with energy logarithmically dependent on their separation, and in the present case the same will be true of the proton vortices, due to the non zero value that is expected [28] for the entrainment factor $\alpha_{\mathrm{n}}^{\mathrm{p}}$ given by (94). The simultaneous presence of neutron and proton vortices can give rise to interesting interaction effects, which have been examined in a Newtonian analysis by the Sedrakians [8]. However the most suitable way to represent these effects explicitly within the present framework is not yet clear to us. 
Another important issue would be to work out the magnetohydrodynamic limit of our equations of motion in order to get rid of superfluous degrees of freedom and to obtain more tractable equations when one wishes to consider some specific problems such as glitches in neutron stars.

\section{Acknowledgements}

We would like to thank L. Lindblom and D. Sedrakian for very stimulating discussions.

\section{References}

[1] D. Langlois, D. Sedrakian, B. Carter, astro-ph/9711042, to be published in Mon. Not. R.A.S (1998)

[2] B. Carter, Class. Quantum Grav. 11, pp 2013-2030 (1994)

[3] B. Carter, D. Langlois, Nuclear Physics B 454, 402 (1995). hep-th/9611082

[4] B. Carter, Int. J. Mod. Phys. 3, pp 15-21 (1994).

[5] G.A. Vardanyan, D.M. Sedrakyan, Sov. Phys. JETP 54, 919 (1981)

[6] G. Mendell, L. Lindblom, Ann. Phys. 205, pp 110-129 (1991).

[7] G. Mendell, Astroph. J 380, pp 515-529 (1991).

[8] A.D. Sedrakian, D.M. Sedrakian, Astroph. J. 447, pp 305-323 (1995).

[9] B. Carter, I.M. Khalatnikov, Ann. Phys. 219, pp 243-265 (1992).

[10] B. Carter, I.M. Khalatnikov, Rev. Math. Phys. 6, pp 277-304 (1994).

[11] D. Pines, M.A. Alpar, Nature 31627 (1985).

[12] B. Carter, H. Quintana, Proc. Roy. Soc. Lond. A331, pp 57-83 (1972).

[13] B. Carter, Commun. Math. Phys. 30, pp 261-286 (1973).

[14] B. Carter, H. Quintana, Astrophys. J. 202, pp 511-522 (1975).

[15] D. Priou, Mon. Not. R.A.S. 254, 435 (1992).

[16] B. Carter, in Relativistic Fluid Dynamics, ed. A. Anile, M. Choquet Bruhat, Lecture Notes in Mathematics 1385, pp 1-64 (Springer -Verlag, Heidelberg, 1989).

[17] M.A. Alpar, P.W. Anderson, D. Pines, J. Shaham, Astroph. J 276, 325 (1984). 
[18] B. Carter, in A random walk in Relativity and Cosmology (Essays in honour of P.C. Vaidya $\&$ A.K. Raychaudhuri), ed. N. Dadhich, J. Krishna Rao, J.V. Narlikar, C.V. Visveshwara, pp 48-62 (Wiley Eastern, Bombay, 1985).

[19] A.F. Andreev, E.P. Bashkin, Sov. Phys. J.E.T.P., 42, 164 (1976).

[20] A.H. Taub, Phys.Rev. 94, 1468 (1954).

[21] B. Carter, Proc. Roy. Soc. Lond. A443, pp 45-62 (1991).

[22] G. Comer, D. Langlois, Class, Quantum Grav. 11, 709 (1994).

[23] I.L. Bekarevich and I.M. Khalatnikov, Sov. Phys. J.E.T.P. 13, 643 (1961)

[24] E.B. Sonin, Rev. Mod. Phys 59, 87 (1987).

[25] L.D. Landau, E.M. Lifshitz, Course of Theoretical Physics 6: Statistical Physics (Pergamon, Oxford, 1959).

[26] B. Carter, D. Langlois, Phys. Rev D51, pp 5855-5864 (1994). hep-th/9507058

[27] D.M. Sedrakyan, K.M. Shakhabasyan, Astrophysics 16, 417 (1980).

[28] M.A. Alpar, S.A. Langer, J.A. Sauls, Astroph. J. 282, 535 (1984).

[29] B. Carter, R. Prix, D. Langlois, "Energy of flux tubes in rotating superconductors", in preparation 